\section{Kawasaki disease presenting with bloody diarrhea and acute renal failure: first case}

\author{
Mary Jacqueline Saviour, Sam Hassan \\ Mediclinic City Hospital, Dubai Health \\ Care City, Dubai, United Arab Emirates
}

\begin{abstract}
Kawasaki disease (KD) is an acute febrile vasculitis of an unknown cause. It affects children $\leq 5$ year of age, even if cases over 5 years old have been also reported. It is the commonest cause of acquired heart diseases in children which may lead to serious morbidity and mortality. The complications and mortality increase when the diagnosis is delayed. One of the main reasons leading to delayed diagnosis and consequent delayed treatment is the unusual presentation of KD. Its unusual manifestations have been increasingly reported to jeopardize the timely diagnosis and proper treatment. As there is not yet available blood test to diagnose it, low threshold should be taken into account for considering $\mathrm{KD}$, when the clinical criteria are not typical. $\mathrm{KD}$ with renal manifestations is infrequently described. We present and discuss a case of an unusual presentation of KD presenting as bloody diarrhea and acute renal failure.
\end{abstract}

\section{Introduction}

Kawasaki disease (KD) is an acute vasculitis disease causing inflammation of medium sized arteries which predominantly affects children less than 5 years of age. Tomisaku Kawasaki first described the disease in 1967.1,2 It is manifested by fever followed by vasodilation and mucosal inflammation. ${ }^{3}$ There are three clinical stages involved in the course of this disease namely acute febrile, subacute and convalescent stages. 4

The acute febrile stage lasts for one to two weeks, with signs and symptoms of fever, conjunctival injection, erythema of the oral mucosa, erythema and swelling of the hands and feet, rash, cervical adenopathy, aseptic meningitis, diarrhea, and hepatic dysfunction. ${ }^{4}$ Myocarditis, pericardial effusion and Coronary arteritis may also be present during this stage, 5 but in general echocardiography usually shows no aneurysms.

The subacute stage commences two weeks after the onset of fever when the rash and lymphadenopathy settle down, while irritability, anorexia, and conjunctival injection continue. In addition to the foresaid signs, fingers and toes appear to desquamate and thrombocytosis occurs. The risk of sudden death is high during this stage due to the development of coronary artery aneurysms. The subacute stage usually lasts for two weeks.

The convalescent stage begins when all clinical signs of illness die out, and continues until the erythrocyte sedimentation rate returns to normal, which lasts six to eight weeks after the onset of illness. ${ }^{4}$

A classic KD can be established by the following diagnostic criteria, five or more days of high fever along with at least four of the remaining five symptoms (bilateral conjunctival hyperemia, ulcerations of the lips and inflammation of the oral cavity, polymorphous rash, edema and desquamation of the extremities and cervical lymphadenopathy) or fever associated with less than 4 of the diagnostic criteria and echocardiographic abnormalities of the coronary arteries.

Kawasaki disease is a systemic vasculitis and can involve multiple organs. Renal manifestations are rarely reported with the exception of sterile pyuria and trace proteinuria. ${ }^{1,3-8}$ We report a case of KD which initially presented with bloody diarrhea and renal failure.

\section{Case Report}

A two and half year old child, with no medical issues in the past was admitted in the hospital with three days history of fever and bloody diarrhea. There was no history of vomiting. On examination child was looking unwell but nontoxic with high fever. He was hyperventilating. His throat was red and congested, left tympanic membrane was red and left upper cervical lymph node was enlarged of $2 \times 1.5 \mathrm{~cm}$ size, firm, tender on palpation. There were signs of dehydration, blood pressure was normal, he was tachycardic and peripheries were cold. There were no signs of meningeal irritation. There were no skin rashes or any changes in the oral mucosa; abdomen was soft with no organomegaly. His height and weight were on the 50th centile.

The initial investigations revealed hemoglobin of $12 \mathrm{~g} / \mathrm{dL}$, white cell count $11,000 / \mathrm{mm}^{3}$ (polymorphs $78 \%$, lymphocyte $20 \%$, and monocytes $2 \%$ ) platelet $120,000 / \mathrm{mm}^{3}$, C-reactive protein $246 \mathrm{mg} / \mathrm{L}$, blood gas analysis revealed metabolic acidosis with respiratory alkalosis and $\mathrm{pH} 7.2$, bicarbonate 13 and $\mathrm{PCo} 2$ 30. Renal biochemistry was altered with sodium
Correspondence: Mary Jacqueline Saviour, Mediclinic City Hospital, Dubai Health Care City, P.O. Box 505004, Dubai, United Arab Emirates.

E-mail: mary.saviour@mediclinic.ae

Key words: Kawasaki disease, hemolytic uremic syndrome, vasculitis, coronary artery aneurysm.

Acknowledgements: the authors are grateful to the parents of the child who allowed to publish this case.

Contributions: the case report was prepared by MJS; SH was involved in the diagnosis and clinical management of the case and reviewing the paper.

Conflict of interest: the authors declare no potential conflict of interest.

Received for publication: 30 March 2017

Revision received: 6 June 2017.

Accepted for publication: 8 June 2017.

This work is licensed under a Creative Commons Attribution NonCommercial 4.0 License (CC BY-NC 4.0).

(C) Copyright M. J. Saviour and S. Hassan, 2017 Licensee PAGEPress, Italy

Pediatric Reports 2017; 9:7163

doi:10.4081/pr.2017.7163

$127 \mathrm{mmol} / \mathrm{L}$, potassium $3 \mathrm{mmol} / \mathrm{L}$, blood urea $13.8 \mathrm{mmol} / \mathrm{L}$ and creatinine 91 $\mathrm{mmol} / \mathrm{L}$. Blood salicylate level was normal and blood ammonia level was also normal. Ultrasound of the neck reported as inflammatory lymphadenopathy.

Initial management was with fluid replacement and empirical antibiotics. The following day after admission, child became oliguric and puffy, urine output decreased despite an adequate amount of fluid administration and the high fever continued. Blood pressure was $99 / 76 \mathrm{mmHg}$ on the 99th centile and he gained $300 \mathrm{~g}$ more of weight.

Urine analysis showed $10-12$ pus cells/HPF and few RBC and urine culture was sterile, Ultrasound scan of the abdomen revealed kidneys of increased echogenicity with no mesenteric lymphadenopathy.

Stool analysis and culture reported as normal. Blood culture showed no organism Mono spot test was negative, throat swab culture was negative and chest $\mathrm{x}$-ray was normal. Lumbar puncture yielded clear fluid with no cells, protein $0.2 \mathrm{~g}$, glucose $3.5 \mathrm{mmol} / \mathrm{L}$.

A repeat biochemistry on third day revealed: Electrolytes sodium of 127 $\mathrm{mmol} / \mathrm{L}$, potassium $5 \mathrm{mmol} / \mathrm{L}$, blood urea 
increased to $15 \mathrm{mmol} / \mathrm{L}$ (normal value 1.2 $6.3 \mathrm{mmol} / \mathrm{L}$ ) and creatinine increased to 117 umol/L (normal value 35-58 umol/L), urine sodium was less than $20 \mathrm{mmol} / \mathrm{L}$ and urine potassium $3.5 \mathrm{mmol} / \mathrm{L}$, urine urea was 137 $\mathrm{mmol} / \mathrm{L}$, urine urea/blood urea 9. Urine osmolality was $347 \mathrm{mos} / \mathrm{L}$, plasma osmolality was 276 . Urine osmolality/plasma osmolality was 1.25 . Urine specific gravity was 1.012 .

On a repeat hemogram, hemoglobin dropped to $10 \mathrm{~g} / \mathrm{dL}$ and platelet count to $100,000 / \mathrm{mm}^{3}$, peripheral blood smear was suggestive of hemolytic picture with fragmented RBC and relatively low platelets. Meanwhile his urine output decreased and he gained weight more of $800 \mathrm{~g}$.

Until this point the overall picture was suggestive of hemolytic uremic syndrome

He was carefully managed with strict input-output chart, daily weight monitoring, and 4-6 hourly BP recording, electrolytes measurements, careful monitoring of fluid balance with fluid restriction to previous days urine output with added insensible fluid loss.

On the fourth day of admission child became very sick, he was looking miserable, conjunctivae became red, developed measles like rash all over the body, lips cracked, tongue and buccal mucosa became intensively red and there was subcutaneous edema over palms and sole.

Based on the foregoing clinical findings, he was diagnosed as Kawasaki disease; intravenous immunoglobulin was given along with oral aspirin- anti-inflammatory dose and supportive management maintaining the fluid and electrolyte balance. There was dramatic improvement clinically. Fever subsided within 24 hours of immunoglobulin infusion and urine output gradually improved and before discharge the renal parameters were returned to normal. He developed peeling of skin around the perianal area. An echocardiography done showed normal anatomy and function of the heart. Subsequently he was discharged on antiplatelet dose of aspirin and advised follow up.

Echocardiography repeated two weeks after discharge showed right main coronary artery aneurysm of $5 \mathrm{~mm}$ in size. Child was continued on antiplatelet dose of aspirin and regular follow up in cardiology outpatient department and an echocardiography which was repeated after a year from the disease onset revealed a normal heart.

\section{Discussion}

Kawasaki disease affects multiple sys- tems of the body, the etiology of which is unknown. Perhaps body's immune reaction to infection along with genetic susceptibility can be attributed to the etiology of $\mathrm{KD}$, as in the case of atopic diseases. ${ }^{9}$ However studies are in the offing on the role of a variety of genes related to inflammation. ${ }^{10}$

It may mimic other systemic disorders as there is multiorgan involvement. Many complications has been described with reference to $\mathrm{KD}^{1}$ and the most important and life threatening among them is the development of coronary artery aneurysm ${ }^{11,12}$ and is the most common cause of acquired heart disease in children in the most developed countries like Japan and USA. ${ }^{11,12}$

However many extra cardiac complications are being reported and renal disease is one among them. It was believed that renal system involvement in KD was uncommon, except findings like sterile pyuria and trace proteinuria. . $^{1,3,8}$

Based on the available information and an exhaustive literature search revealed that the, complications like prerenal acute kidney injury (AKI), renal acute kidney injury caused by tubulointerstial nephritis, immune complex mediated nephropathy, acute nephritic syndrome, renal tubular abnormalities, renal abnormalities in imaging studies, and renal artery lesions are increasingly being recognized in KD. 13 Only two cases of HUS has been reported in association with KD. ${ }^{14,15}$ Ferriero and Wolfsdorf 14 reported for a two year old girl with KD, who recovered with supportive care only and Hedrich et al reported a three year old girl with KD who developed HUS and Henoch schonlein purpura. ${ }^{15}$ We report a case of $\mathrm{KD}$ which presented initially as bloody diarrhea and renal failure. Luckily the patient showed typical features of KD on the $4^{\text {th }}$ day of admission. On the contrary if the patient had not shown these features, there would have been a delay in the diagnosis and treatment.

Hemolytic uremic syndrome (HUS) is established as a syndrome of heterogeneous group of correlative entities since 1955. In children it is considered as a predominant cause of community acquired acute renal failure. Statistics disclose that the probability of occurrence of HUS is one to three cases per 100,000 children per year. 16 The three intrinsic features of HUS are microangiopathic hemolytic anemia, thrombocytopenia, and renal insufficiency. ${ }^{16}$

The etiologies of HUS are mainly classified as infection-induced, medicationinduced, familial or genetic and allied with systemic diseases. ${ }^{17}$

HUS combined with diarrhea; the so called D+ HUS is the most common form, which manifests as a sudden onset of hemolytic anemia, thrombocytopenia, and acute renal failure preceded by gastrointestinal enteritis. The other types of HUS are often expressed as atypical HUS or DHUS (HUS without diarrhea).

The renal impairment includes renal microangiopathy expressed as a reduction in glomerular filtration rate, proteinuria and hematuria. ${ }^{18}$ Microvascular injury with endothelial cell damage is supposed to be the pathology of all forms of HUS. ${ }^{17}$ The etiology of HUS can be categorized as stimulation by infection, medication, genetic, and in connection with systemic diseases characterized by microvascular injury. ${ }^{17}$

The characteristic features are separation of the endothelial cells from the basement membrane and swelling of the sub endothelial space leading to the occlusion of the capillary lumen. ${ }^{19}$ If it becomes severe, the injury can progress to arterioles and small arteries which is accompanied by the formation of micro thrombi. 20

This process can also occur outside the kidney, in organs such as heart and brain. ${ }^{21}$

Laboratory findings include microangiopathic hemolytic anemia with findings on the peripheral blood smear (i.e. Schistocytes/helmet cells, and echinocytes) along with findings of acute renal injury (hematuria, proteinuria, or elevated creatinine level). Thrombocytopenia is usually also detected early in the illness but subsequently the platelet count can become normal or even elevated. Other findings in HUS include a negative Combs' test in addition to evidence of hemolysis like an elevated LDH, elevated reticulocyte count, low serum haptoglobin, and an unconjugated hyperbilirubinemia. Coagulation tests are usually normal. ${ }^{17}$

Our case had all the telltale signs and symptoms of KD which developed on the 6th day of illness, fever for more than 5 days, erythema of lips oral mucous membrane (Figure 1A), left cervical lymph node enlargement, strawberry tongue, edema of hands and feet (Figure 1B and C), erythematous rashes on the body, skin peeling around the perianal area (Figure 1D), redness of eyes and definite cardiac involvement (Figure 2).

Biochemistry revealed elevation of blood urea and creatinine and hyponatremia. Urine analysis showed white blood cells and red blood cells and urine culture showed no growth.

A repeated hemogram showed drop in hemoglobin and thrombocytopenia instead of thrombocytosis which happens in KD. And blood smear examination showed hemolytic picture with fragmented RBC and relative thrombocytopenia. Even though Kawasaki disease shows thrombo- 
cytosis in the acute stage, our case showed thrombocytopenia.

The exact mechanism of renal damage in $\mathrm{KD}$ is unknown as in most cases renal biopsy was not done and hence the histopathology could not be made out.

A renal biopsy was not done for the two reported cases of $\mathrm{KD}$ associated with HUS, 14,15 mentioned earlier as in our case report, It may be inferred that the vasculitis associated with $\mathrm{KD}$, probably have involved the kidney to produce endothelial damage in the renal and glomerular endothelium, eventually resulting a clinical picture of HUS. 14

This is supported by the fact that features of HUS occurred during the initial period of his illness in which stage 1 of vasculitis of Kawasaki diseases is found and all the renal manifestations resolved along with the signs and symptoms of KD.

With the increased use of intravenous immunoglobulin (IVIG), there is a little but definite risk of kidney damage, ${ }^{22}$ but in our case the features of hemolytic uremic syndrome were seen before the infusion of IVIG.

Kawasaki syndrome associated with Yersinia infection presents as abdominal pain, diarrhea, fever, skin rash, conjunctivitis, erythema nodosa and lymphadenopathy and closely mimics KD. ${ }^{14,22}$ Our case did not show any mesenteric lymphadenopathy which is characteristic of Yersinia infection and his stool culture was negative. ${ }^{23,24}$

Pre-renal cause of renal failure could be excluded by the renal profile and urinary studies. urine osmolality 347 , urine specific gravity 1.012, urine urea/blood urea was 9, urine osmolality/plasma osmolality 1.25 all of which indicates intrinsic cause of renal failure.

The initial clinical features of high fever with bloody diarrhea, thrombocytopenia, drop in hemoglobin and renal failure are clinical features of HUS, although the parameters like platelets and hemoglobin did not show a significant drop. Even though hemolytic uremic syndrome was kept as the first differential diagnosis, all the typical features of HUS were not present in our case. While a peripheral smear showed fragmented RBC, other laboratory features suggestive of hemolysis like Coombs test, LDH level, reticulocyte count, serum haptoglobulin and a liver function test were unfortunately not done in our case.

Stool culture may be positive for E Coli 0157, H7 in D+HUS, however negative culture will not exclude HUS or 0157 , and $\mathrm{H} 7$ infection. ${ }^{25}$ Since the clinical featured of HUS vividly cited were not present in the case, it is not being presented as HUS complicating $\mathrm{KD}$, but as $\mathrm{KD}$ presenting as
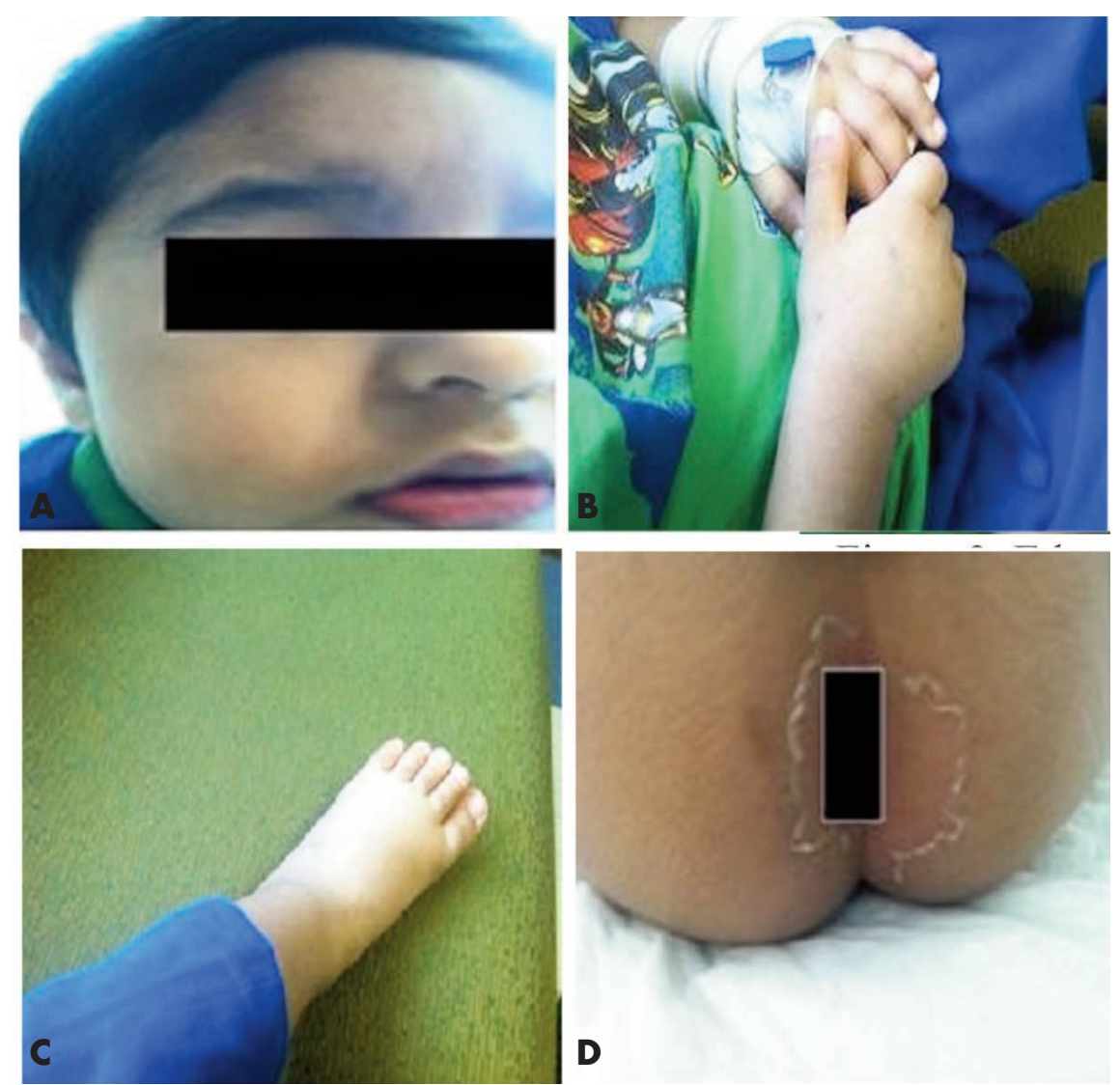

Figure 1. A) Erythema of lips; B) edema of hands; C) edema of feet; D) skin peeling around perianal area.

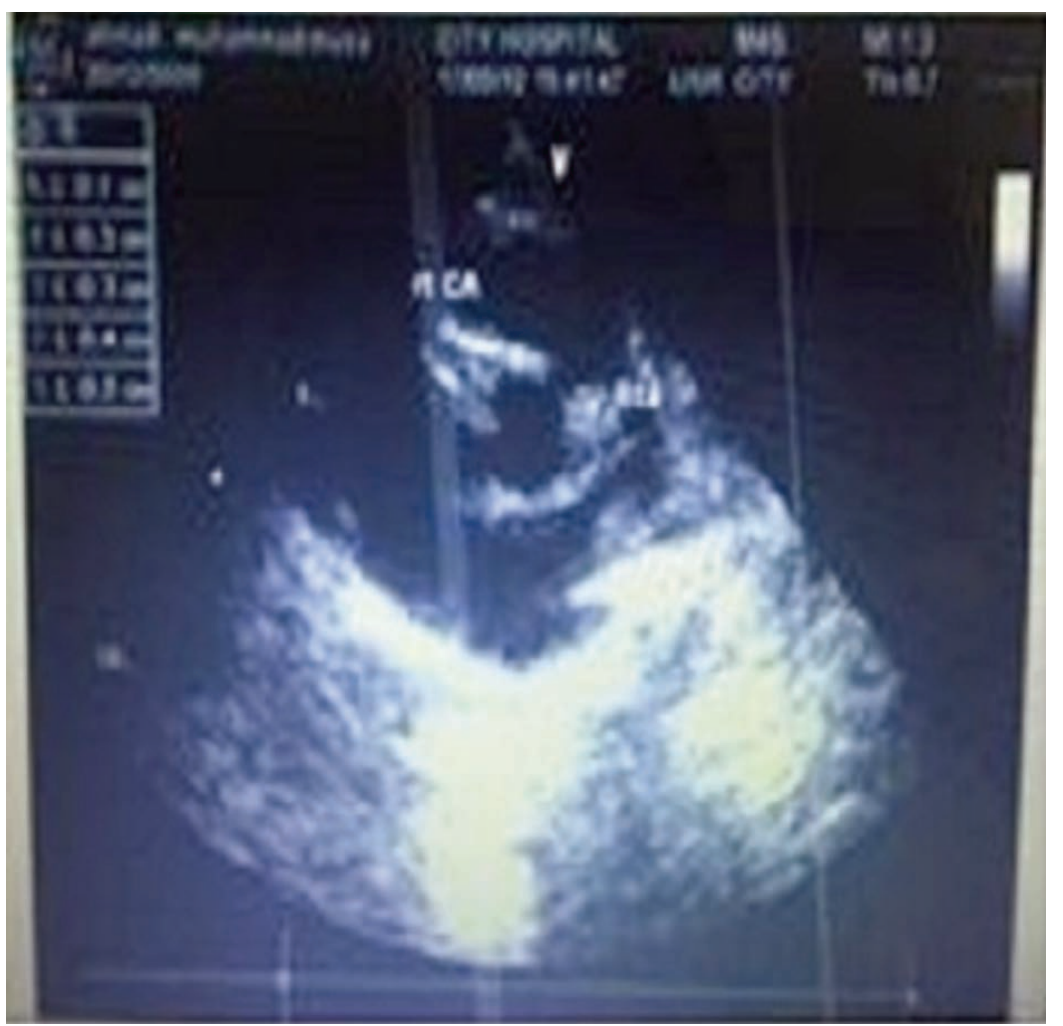

Figure 2. Echo cardiograph. 
bloody diarrhea and renal failure. In our case, adequate fluid was administered as the child was having dehydration from loose stools. In spite of that he developed oliguria and became puffy.

\section{Conclusions}

Medical practitioners often face challenges in timely diagnosis of KD due to the atypical presentation of the disease. The establishment of a definitive diagnosis may not be possible upon initial presentation. The association of kidney and urinary tract is rare in Kawasaki disease. To the best of our knowledge, our case is the first report of its kind in literature in which Kawasaki Disease presenting as bloody diarrhea and renal failure. This reporting of unusual presentation should be of help to other pediatricians for the timely diagnosis and appropriate management.

\section{References}

1. Kawasaki T, Kosaki F, Okawa S, et al. A new infantile acute febrile mucocutaneous lymph node syndrome (MLNS) prevailing in Japan. Pediatrics 1974;54:271-6.

2. Burns JC, Glodé MP. Kawasaki syndrome. Lancet 2004;364:533-44.

3. Melish ME, Hicks R, Larson EJ. Mucocutaneous lymph node syndrome in the United States. Am J Dis Child 1976;130:599-607.

4. Castro PA, Urbano LM, Costa IM. [Kawasaki disease]. An Bras Dermatol 2009;84:317-29. [Article in Portuguese].
5. Rowley AH, Shulman ST. Kawasaki syndrome. Clin Microbiol Rev 1998;11:40514.

6. Hirose O, Misawa H, Kijima Y, et al. [Two-dimensional echocardiography of coronary artery in Kawasaki disease (MCLS): detection, changes in acute phase, and follow-up observation of the aneurysm]. J Cardiogr 1991;11:89-104. [Article in Japanese]

7. Kawasaki T. Acute febrile mucocutaneous syndrome with lymphoid involvement with specific desquamation of the fingers and toes in children. Arerugi 1967;16:178-222. [Article in Japanese].

8. Burns JC. Kawasaki disease. Adv Pediatr 2001;48:157-17.

9. Leonardi S, Miraglia del Giudice M, La Rosa M, Bellanti J. Atopic disease, immune system and environment. Allergy Asthma Proc 2007;28:410-7.

10. Onoyama S, Ihara K, Yamaguchi Y, et al. Genetic susceptibility to Kawasaki disease: analysis of pattern recognition receptor genes. Hum Immunol 2012;73:654-60.

11. Scuccimarri R. Kawasaki disease. Pediatr Clin North Am 2012;59:425-45.

12. Son MB, Newburger JW. Kawasaki disease. In: Kliegman RM, Stanton BF, Geme JWSt III, et al., eds. Nelson textbook of pediatrics. 19th ed. Philadelphia: Saunders; 2011.

13. Watanabe T. Kidney and urinary tract involvement in Kawasaki Disease. Int J Pediatr 2013;2013:831834.

14. Ferriero DM, Wolfsdorf JI. Hemolytic uremic syndrome associated with Kawasaki disease. Pediatrics 1981;68:405-6.

15. Heldrich FJ, Jodorkovsky RA, Lake AM, Parnes CA. Kawasaki syndrome:
HUS and HSP complicating its course and management. Maryland Med J 1987;36:7646.

16. Razzaq S. Hemolytic-uremic syndrome: an emerging health risk. Am Fam Physician 2006;74:991-6.

17. Van Why S, Avner E. Hemolytic-uremic syndrome. In: Kliegman RM, Stanton BF, Geme JWSt III, et al., eds. Nelson textbook of pediatrics. 19th ed. Philadelphia: Saunders; 2011.17911794.

18. Kaplan BS, Drummond KN. The hemolytic uremic syndrome is a syndrome. N Engl J Med 1978;298:964-6.

19. Goldstein MH, Churg J, Strauss L, et al. Hemolytic-uremic syndrome. Nephron 1979;23:263-72.

20. Vitsky BH, Suzuki Y, Strauss L, et al. The hemolytic uremic syndrome: a study of renal pathologic alterations. Am J Pathol 1969;57:627-47.

21. Upadhyaya K, Barurck K, Fishaut M, et al. The importance of nonrenal involvement in hemolytic-uremic syndrome. Pediatrics 1980;65:115-20.

22. Fakhouri F. Intravenous immunoglobulins and acute renal failure: mechanism and prevention. Rev Med Interne 2007;28:4-6.

23. Abe J, Onimaru M, Matsumoto S, et al. Clinical role for a super antigen in Yersinia pseudo tuberculosis infection. J Clin Invest 1997;99:1823-30.

24. Takeda N, Usami I, Fujita A, et al. Renal complications of Yersinia pseudo tuberculosis infection in children. Contr Microbiol Immunol 1991;12:301-6.

25. Rowe PC. Escherichia coli O157:H7, other verotoxin-producing $\mathrm{E}$ coli and the hemolytic uremic syndrome in childhood. Can J Infect Dis 1995;6:10510 . 